\title{
Bioengineered Silver Nanoparticle from Spirulina platensis in Attenuating Biofilm Mediated Virulence in Vibrio parahemolyticus: An in vitro and in vivo Approach
}

\author{
Rathna Kumarasamy*, Padavala Navyaka, Ekramul Haque \\ Department of Microbiology, School of Life Sciences, Pondicherry University, Puducherry, INDIA.
}

\begin{abstract}
Objectives: In this study, bioengineered AgNPs (silver nanoparticles) from Spirulina platensis was used to counter the biofilm mediated virulence by Vibrio parahemolyticus. Methods: AgNPs were synthesized from crude Spirulina platensis extract and characterized using UV spectrophotometry, Transmission electron microscope (TEM). Antibacterial effect against aquatic pathogen Vibrio parahemolyticus was carried out. Further, the nanoparticle at sub MIC (minimum inhibitory concentration) level were screened for anti-virulence property under in vitro studies which include biofilm inhibition using microtiter plate assay, microscopic analysis under light microscope and SEM (Scanning electron microscope) imaging, EPS (Exopolysaccharide) inhibition, CSH(Cell surface hydrophobicity) inhibition using BATH(Bacterial adhesion to hydrocarbons) assay, swarming and swimming motility inhibition, growth pattern analysis followed by in vivo study in aquatic animal model brine shrimp and its survival percentage along with light microscopic imaging was performed. Results: AgNPs, at $320 \mu \mathrm{g} / \mathrm{ml}$ showed bactericidal activity, minimal inhibitory concentration was found to be $160 \mu \mathrm{g} / \mathrm{ml}$. All anti-virulence assay performed at below sub MIC concentration. Biofilm and EPS showed 63\% and 53\% inhibition
\end{abstract}

respectively, CSH was found to be decreased 3-fold. Growth pattern at sub MIC compared with non-treated showed similar result. In vivo study increased survival percentage (90\%) when treated with AgNPs, compared to untreated $(20 \%)$. A 7 -fold increased resistance to pathogen was found and light microscope also confirms the efficacy of $\mathrm{Ag}$ nanoparticle. Conclusion: AgNPs become a substitute to the current commercial antibiotics used in aquaculture against Vibriosis, by functioning as an efficient quorum sensing inhibitor.

Keywords: AgNPs, Vibrio parahemolyticus, Anti-virulence, Biofilm, Survival rate.

\section{Correspondence}

K Rathna

Research Scholar, Department of Microbiology, School of Life Sciences,

Pondicherry University, Puducherry-605014, INDIA.

Phone no: +919043167875

Email: rathnamrks13@gmail.com

DOI: 10.5330/ijpi.2020.4.85

\section{INTRODUCTION}

Nanotechnology has been a promising approach in the field of research and its wide application in multidisciplinary field has led to its popularity. ${ }^{1}$ The particularly antimicrobial property of the silver nanoparticle had its enormous application in medicine, animal husbandry, packaging, cosmetics. ${ }^{2}$ Most of the research focuses on the inhibitory effect of silver nanoparticles Against different pathogens such as Escherichia coli, Vibrio cholera, Pseudomonas aeruginosa and Staphylococcus aureus. ${ }^{3}$ But indeed, there is a big gap in the study of the antagonistic effect of the silver nanoparticle on the virulence factors of pathogenic micro-organisms. Nanoparticles can be synthesized by several methods, there is always a need to follow a green path that safely resonates the synthesis of nanoparticles through micro-organisms, algae and plants, which is considered a safe method for the environment, profitable, clean and efficient. ${ }^{4}$ Among micro-organism cyanobacteria which possess an sustainable resource for various bioactive metabolic products and possess a high therapeutical application, ${ }^{5}$ one such is Spirulina platensis which is well known for several pharmacological activities such as antimicrobial, anticancer, metalloprotective as well as immunostimulant and antioxidant. Since natural products like phenols, PUFA, Pyocyanin and other micro and macro nutrients are highly enriched in Spirulina, it showed antimicrobial activity against several pathogen. ${ }^{6}$ Therefore, in the study Ag nanoparticle bioengineered from Spirulina platensis was used.

Vibrio parahemolyticus is one of the common food borne pathogen primarily associated with seafood that is widely distributed in the marine estuarine environment and acts as a multivirulent pathogenic host for humans as well as aquatic animals. ${ }^{7}$ Recent reports point out that aquatic pathogens are becoming increasingly resistant to most existing antibiotics, mainly due to the inadequate disposal of antibiotics, disinfectants and derivatives by industries and the overuse of antibiotics had led to the evolution and emergence of antibiotic resistant strains specifically in an aqueous environment. Due to the ineffectiveness of current chemotherapies, an alternative approach was therefore Ag nanoparticle, A cost effective drug that showed a broad antibacterial spectrum against most aquatic pathogen. ${ }^{8}$ Several studies have reported the antimicrobial properties of silver nanoparticles, but only Some research has been reported on the drug's efficacy against the virulence traits of the pathogen, which is very essential for controlling the pathogen in the aquatic environment and also contributes to a safer use of nanoparticle as a drug. Hence current study focuses on Ag nanoparticle efficacy in controlling the virulence cascade in pathogenic vibrio.

\section{MATERIALS AND METHODS}

\section{Spirulina biomass production and extraction preparation}

Spirulina platensis was grown in Zarrouck Media under laboratory condition at $28^{\circ} \mathrm{C}, \mathrm{pH} 9$, with $16 / 8$ light/dark period for 12 days. After which the culture was harvested and washed thrice with distilled 
water, followed by shade drying for 4 days and ground to fine powder and Soxhlet extracted with methanol which was further concentrated to get a crude residue. The dried extract was dissolved in DMSO and used for silver nanoparticle synthesis.

\section{Synthesis of Ag nanoparticles}

In present study $\mathrm{Ag}$ nanoparticle was synthesized according to Kannan et al. ${ }^{9} 2 \mathrm{ml}$ of spirulina extract was added to $1 \mathrm{mM}$ of $100 \mathrm{ml}$ of silver nitrate solution in $250 \mathrm{ml}$ Erlenmeyer flask. The change in the color of the solution to brown color indicate the nanoparticle synthesis and the solution also monitored in UV spectrophotometer. The solution was then centrifuged at $15000 \mathrm{rpm}$ for $20 \mathrm{~min}$ supernatant was discarded and bottom pellet was repeatedly rinsed with sterile distilled water to remove impurities and are stored.

\section{Characterization of Ag nanoparticle}

Ag nanoparticle were characterized using various instrumentation analyses. The formation of Ag nanoparticle was analyzed in Shimadzu scanning spectrophotometer in a range of 200 to $800 \mathrm{~nm}$ wavelength for a Plasmon resonance peak. The morphological analysis was done using High resolution Transmission Electron Microscope (HRTEM) M (JEOL JEM-1400 Orius SC600A camera with digital micrograph) at an accelerating voltage of $120 \mathrm{kV}$. The grid was scanned and the crystal structure of the $\mathrm{g} / \mathrm{ml}$ was determined using the selected area electron diffraction (SAED) pattern. Crystal structure further confirmed using Scanning Transmission Electron Microscope (STEM).

\section{Bacterial strain and growth condition}

Vibrio parahemolyticus, an aquatic bacterial pathogen was used. The strain was grown in Luria Bertani broth supplemented with $2 \% \mathrm{NaCl}$ at $30^{\circ} \mathrm{C}$. For experiments $\mathrm{OD}$ of 0.4 at $600 \mathrm{~nm}$ were used.

\section{Antimicrobial, Minimal inhibitory concentration (MIC), Minimal bactericidal concentration (MBC)}

Antimicrobial activity was performed in Luria Bertani Agar were the diluted bacterial cell were swabbed onto plate and wells were made, Ag nanoparticle suspension was added and incubated for $24 \mathrm{hr}$ for clear zone of inhibition.

MIC and MBC determined according to Humphries et al. ${ }^{10} \mathrm{Ag}$ nanoparticle Stock solution of $500 \mu \mathrm{g} / \mathrm{ml}$ was prepared and serially diluted in microtiter plate and bacterial cells of $106 \mathrm{CFU} / \mathrm{ml}$ were added with $30 \mu \mathrm{l}$ of resazurin and incubated for $24 \mathrm{hr}$. Blue color indicates the no bacterial growth and pink / colorless indicate bacterial growth, further each diluted sample in microtiter plate were plated on Muller Hinton Agar plate and incubated for $24 \mathrm{hr}$ to determine the MBC.

\section{Effect of Ag nanoparticle on biofilm formation}

\section{Microtiter plate assay}

Quantification of biofilm performed according to Packiavathy et al. ${ }^{11}$ The 96 well plate with Ag nanoparticle at different concentration was added to Vibrio parahemolyticus strain along with control without Ag nanoparticle and then the plates were incubated for $24 \mathrm{hr}$ and the media was discarded and rinsed with sterile distilled water followed by staining with $0.4 \%$ crystal violet, excess stain was rinsed, air dried and the stained cells were solubilized in $20 \%$ glacial acetic acid. Absorbance measured at $570 \mathrm{~nm}$. Percentage biofilm inhibition calculated using formula

Percentage of inhibition $=[($ Control OD - Test OD $) /$ Control OD $] \times 100$

\section{Microscopic analysis}

Luria Bertani broth with $2 \% \mathrm{NaCl}$ was added to 6 well microtiter plate and glass cover slip along with Vibrio parahemolyticus strain and Ag nanoparticles were added and incubated for $24 \mathrm{hr}$. For light microscope analysis the glass coverslip was stained with $0.4 \%$ crystal violet and excess stain was rinsed with sterile distilled water and observed under light microscope at $40 \mathrm{x}$ magnification according to Sarkar et al. ${ }^{12}$ and for SEM analysis the coverslip was completely air dried for $2 \mathrm{hr}$ and observed under scanning electron microscope.

\section{Effect of Ag nanoparticle on exopolysaccharide}

Eps secretion by Vibrio parahemolyticus was estimated according to Gowrishankar et al. ${ }^{13}$ in which Vibrio parahemolyticus inoculated in Luria Bertani broth with $2 \% \mathrm{NaCl}$ in 6 well microtiter plate was added along with cover slip and incubated for $24 \mathrm{hr}$, the coverslip was gently removed and rinsed in $0.9 \% \mathrm{NaCl}$, the suspended cell in $\mathrm{NaCl}$ were added with equal amount of $5 \%$ phenol and 5 volumes of con h2so 4 and incubated in dark for $1 \mathrm{hr}$ was further centrifuged at $10000 \mathrm{rpm} 10 \mathrm{~min}$ and od taken at $490 \mathrm{~nm}$.

\section{Effect of Ag nanoparticle on cell surface hydrophobicity}

Bacterial adhesion to hydrocarbon (BATH) assay was performed based on Courtney et al. ${ }^{14} \mathrm{Ag}$ nanoparticle treated and non-treated $V$. parahemolyticus strain was added with equal volume of toluene and vortexed for $2 \mathrm{~min}$ and kept undisturbed for $10 \mathrm{~min}$ at room temperature and O.D of aqueous phase was taken at $600 \mathrm{~nm}$. The percentage of hydrophobicity calculated using Hydrophobicity (\%) $=[1$ (Final OD after vortexing/Initial OD before vortexing)] $\times 100$.

\section{Effect of Ag nanoparticle on motility}

Swimming motility was performed according to Whitaker et al. ${ }^{15}$ Luria Bertani broth with $2 \% \mathrm{NaCl}$ of $0.3 \%$ Agar with $\mathrm{Ag}$ nanoparticle and control without $\mathrm{Ag}$ nanoparticle and the pathogen was gently stabbed into medium and incubated at $30^{\circ} \mathrm{C}$ for $24 \mathrm{hr}$. Swarming motility in which Luria broth with $2 \% \mathrm{NaCl}$ of $0.5 \%$ Agar was added and the pathogen was spot inoculated in the surface of medium and incubated for $60 \mathrm{hr}$ at $30^{\circ} \mathrm{C}$.

\section{Growth pattern analysis}

In order to confirm the test sample Ag nanoparticle at concentration is non-bactericidal, growth analysis was done with the $1 \%$ Vibrio parahemolyticus, added to Luria Bertani broth with $2 \% \mathrm{NaCl}$ supplemented with Ag nanoparticle at concentration $80 \mu \mathrm{g} / \mathrm{ml}$ and absorbance was recorded for every 1-hr interval for $24 \mathrm{hr}$. The assay was performed in triplicate along with controls.

\section{In vivo efficacy study in aquatic animal model Brine shrimp}

Brine shrimp cysts were hydrated in 1-liter seawater with salinity of $35 \mathrm{ppt}$ at a maximum aeration. After $24 \mathrm{hr}$ the hatched larvae were transferred to fresh sea water and feeded with soyabean powder. ${ }^{16}$ After which 5 days old larvae were used to conduct the experiment followed according to Brackman et al. ${ }^{17}$ Experiments were divided into four groups, group1-Artemia treated with Ag nanoparticle along with Vibrio parahemolyicus, group 2-Artemia with Vibrio parahemolyticus as positive control, group 3- Artemia with Ag nanoparticle without pathogen to check its toxicity and group 4-Artemia with only larvae, as a control. Survival percentage was calculated till $48 \mathrm{hr}$ according to Packiavathy et al. ${ }^{18}$ using the following formula

Survival percentage $=[$ (number of mortality/numbers of survival $)$ $\times 100)]$. 


\section{Statistical analysis}

All the experiments were performed in triplicates and the values were given as mean \pm standard deviation (SD) using Microsoft Excel 2010 (Microsoft, USA)

\section{RESULTS}

\section{Characterization of Ag nanoparticle}

Ag nanoparticle synthesized was initially confirmed using the color change from yellow to brown, where the silver nitrate solution is reduced to silver nanoparticle which was further confirmed using UV visible spectroscopy, a plasmon resonance peak was observed at $420 \mathrm{~nm}$ (Figure 1) Further morphology of Ag nanoparticle was studied using HRTEM for further confirmation of Ag nanoparticles size and shape, the obtained silver nanoparticles found spherical in shape and these nanoparticles were formed as clear dispersals with few Agglomerated and micrograph which showed the presence of interplanar spacing lattice fringes The average size of green synthesized Ag nanoparticle was found to be $13 \mathrm{~nm}$ (Figure 2). The selected area electron diffraction (SAED) in which the clear specific rings were spotted and it was assigned to the presence of $\mathrm{Ag} 111,220$ and 222 planes of the face-centered cubic structured (FCC) Ag nanoparticles obtained reveal the good crystalline nature of nanoparticle and it was confirmed that, these are green route mediated synthesis of AgNPs. STEM image also additionally confirms the Ag nanoparticle (Figure 3).

\section{Bactericidal and MIC, MBC concentration}

Antimicrobial activity of synthesized nanoparticle against Vibrio parahemolyticus showed a bactericidal activity at $320 \mu \mathrm{g} / \mathrm{ml}$ concentration and MIC was found to be $160 \mu \mathrm{g} / \mathrm{ml}$ (Figure 4). Thus, in order to perform anti-virulence assay sub MIC concentrations and below sub MIC concentrations were used which does not interfere in bacterial growth.

\section{EPS and CSH Virulence inhibition}

Exopolysaccharide is secreted by bacteria which form a gel like matrix which help in biofilm formation in host, an important criterion in invading the host. On treatment with Ag nanoparticle at low sub MIC

A

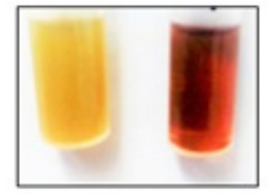

B

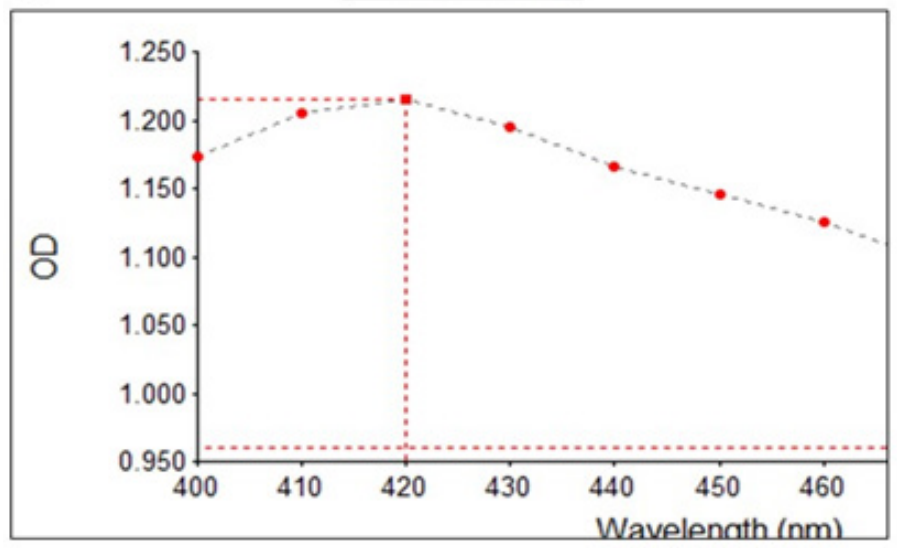

Figure 1: (A) visible color change indicating the synthesis of Ag nanoparticles. (B) UV-Vis analysis of the synthesized nanoparticles showing a peak at $420 \mathrm{~nm}$ indicating the formation of nanoparticles. concentration at $(40,60,80) \mu \mathrm{g}$ it showed a noticeable inhibition up to $53 \%$ at $80 \mu \mathrm{g}$ (Figure 5). Cell surface hydrophobicity, which is key phenomenon in initial attachment to host was also decreased up to 3 -fold at $80 \mu \mathrm{g} / \mathrm{ml}$ (Figure 6).

\section{Biofilm inhibition}

Biofilm is a key unique architecture formed by bacteria which involve several quorum sensing signals in construction and provide role in resistance to several drugs and antibiotics. In current study $\mathrm{Ag}$ nanoparticle at sub MIC concentration $80 \mu \mathrm{g} / \mathrm{ml}$ showed a maximum of $63 \%$ inhibition (Figure 7). Microscopic analysis was performed using light and scanning electron microscope to study the alteration in biofilm matrix results show that thick biofilm formation in control compared to treated were the disintegrated and dispersed microcolonies are visible
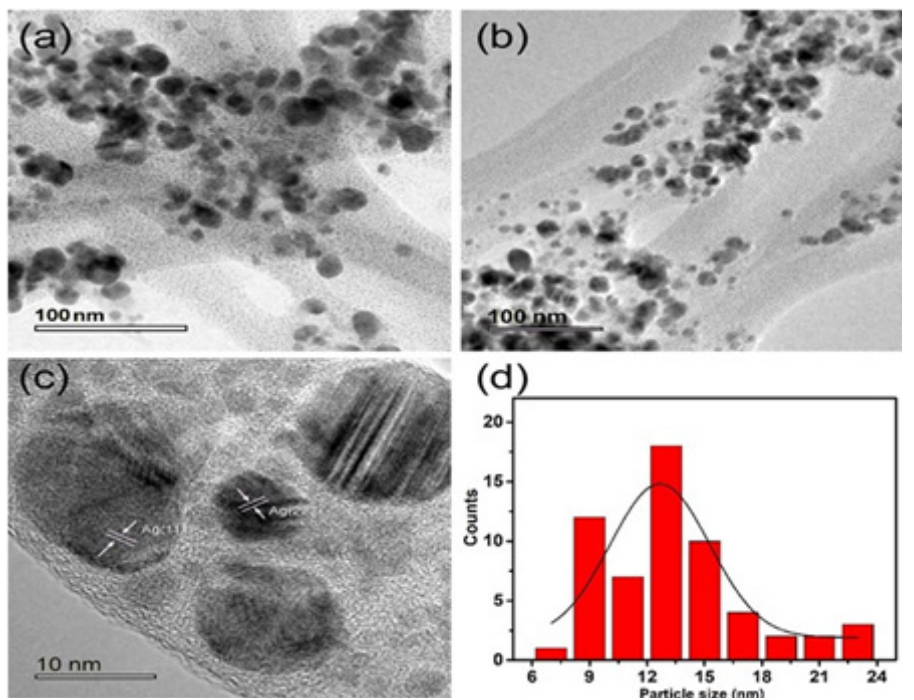

Figure 2: HR-TEM analysis of Ag nanoparticles demonstrating spherical in shape at different magnifications (a)(b)100nm (c) $10 \mathrm{~nm}$ (d) average size ranging from $12-13 \mathrm{~nm}$.
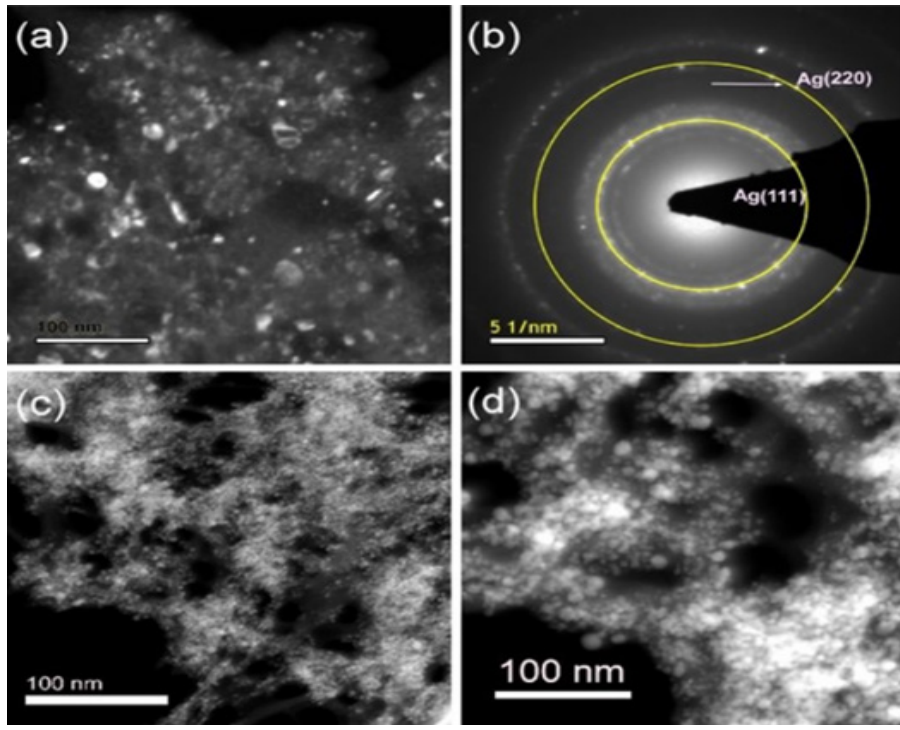

Figure 3: (a) Dark field imaging of the Ag nanoparticles at 100nm magnification (b) SAED analyze the Electron diffraction pattern of ag nanoparticles showing respective crystal planes representing of FCC structure and (c, d) STEM images of the nanoparticles show the crystal structure of $\mathrm{Ag}$ nanoparticles. 
which confirm the disruptive nature of $\mathrm{Ag}$ nanoparticle shown in (Figure 8).

\section{Motility inhibition}

Motility also play a role in biofilm formation, it is very much essential for pathogen to vast spread in host surface, current study Ag nanoparticle showed a inhibition against swimming and swarming motility at $80 \mu \mathrm{g} /$ $\mathrm{ml}$ (Figure 9).

\section{Analysis of growth pattern}

Growth pattern was studied at sub MIC and compared with control, which confirm that sub MIC concentration do not interfere in growth pattern shown in (Figure 10).

\section{In vivo efficacy study, survival rate}

Brine shrimp, aquatic model was treated with sub MIC $80 \mu \mathrm{g} / \mathrm{ml}$ and survival rate on challenge with Vibrio parahemolyticus shows that 7-fold
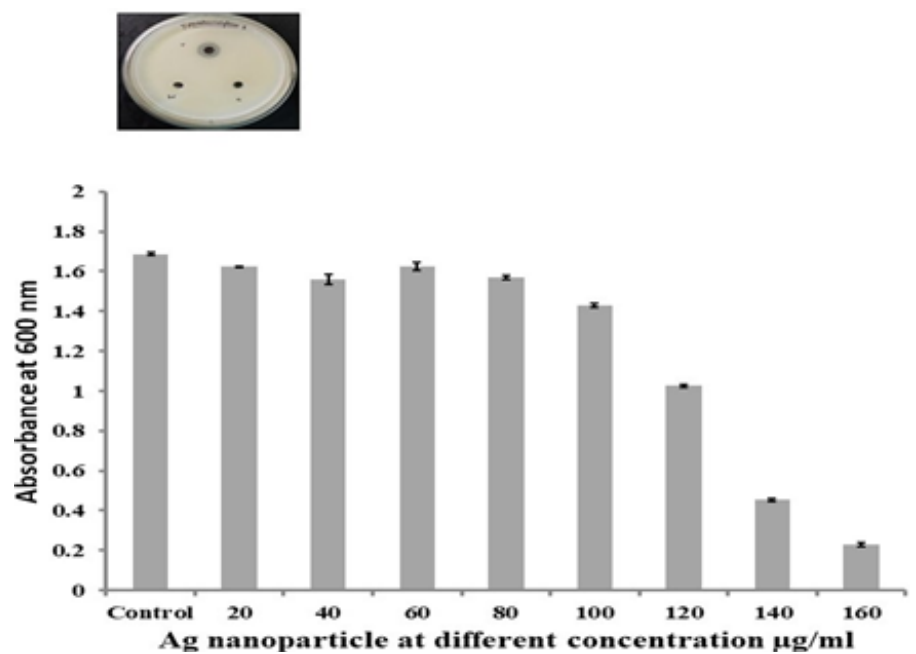

Figure 4: Antimicrobial activity of ag nanoparticles synthesized from Spirulina platensis showing clear zones of inhibition against the pathogenic shrimp Vibrio parahemolyticus and graph represents its Inhibitory effect at different concentration. All the experiments were performed in triplicates and the values were given as mean \pm standard deviation $(n=3)$.

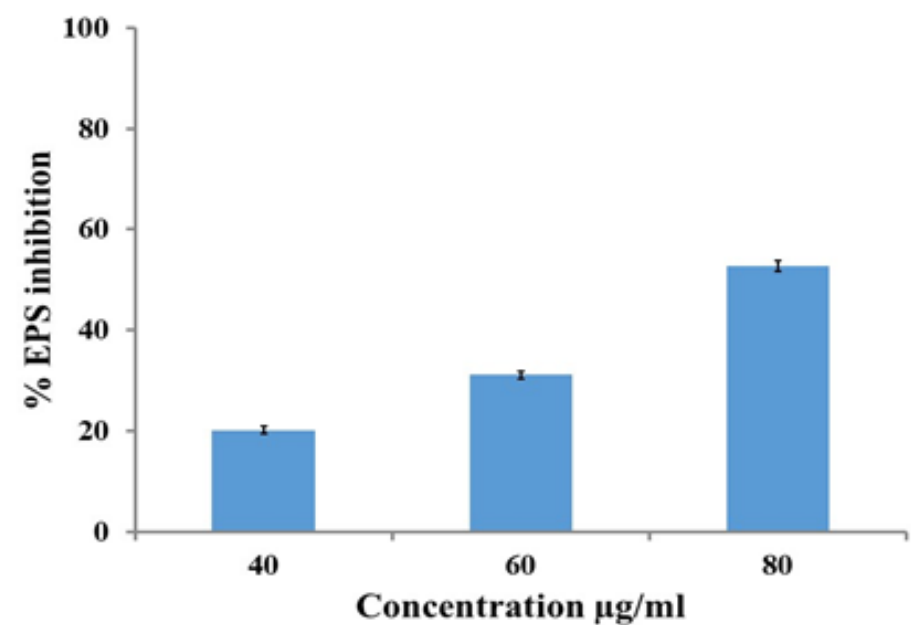

Figure 5: Percentage EPS inhibition of Vibrio parahemolyticus at different concentration of $\mathrm{Ag}$ nanoparticle. All the experiments were performed in triplicates and the values were given as mean \pm standard deviation $(n=3)$. increase in survival (Figure 11). Microscopic observation under 45x magnification shows the Brine shrimp untreated with Ag nanoparticle were easily infected became fragile and entire organism got collapsed whereas the Ag nanoparticle treated showed a drastic difference and resistance to pathogen from being infected (Figure 12).

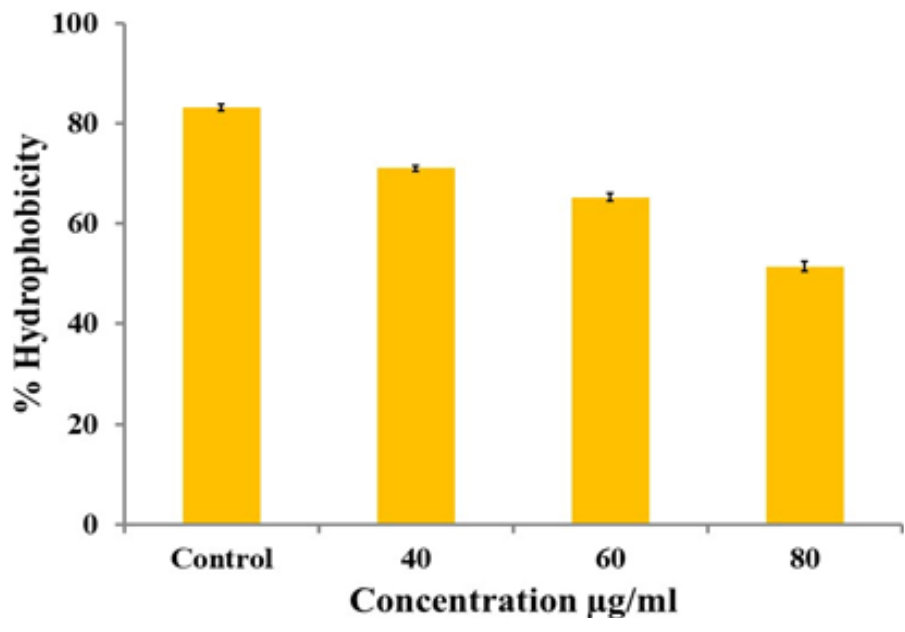

Figure 6: Percentage cell surface hydrophobicity of Vibrio parahemolyticus at different concentration of ag nanoparticle. All the experiments were performed in triplicates and the values were given as mean \pm standard deviation $(n=3)$.

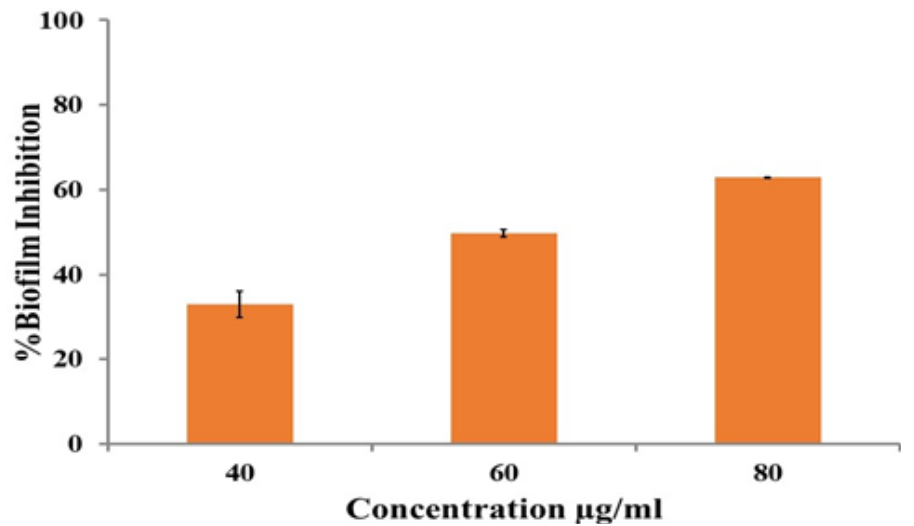

Figure 7: Percentage biofilm inhibition of Vibrio parahemolyticus at different concentration of $\mathrm{Ag}$ nanoparticle. All the experiments were performed in triplicates and the values were given as mean \pm standard deviation $(n=3)$.
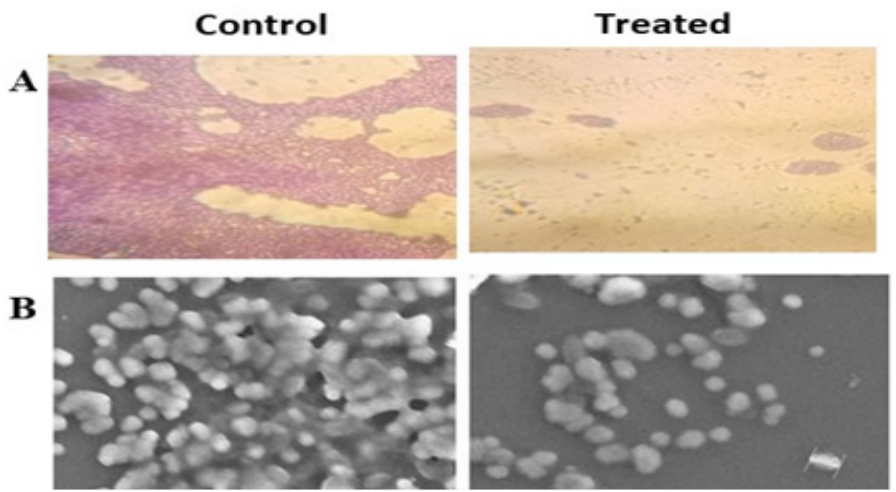

Figure 8: Microscopic visualization of biofilm formation and disintegration of Vibrio parahemolyticus on treatment with ag nanoparticle under (A). Light microscope and (B) scanning electron microscope. 


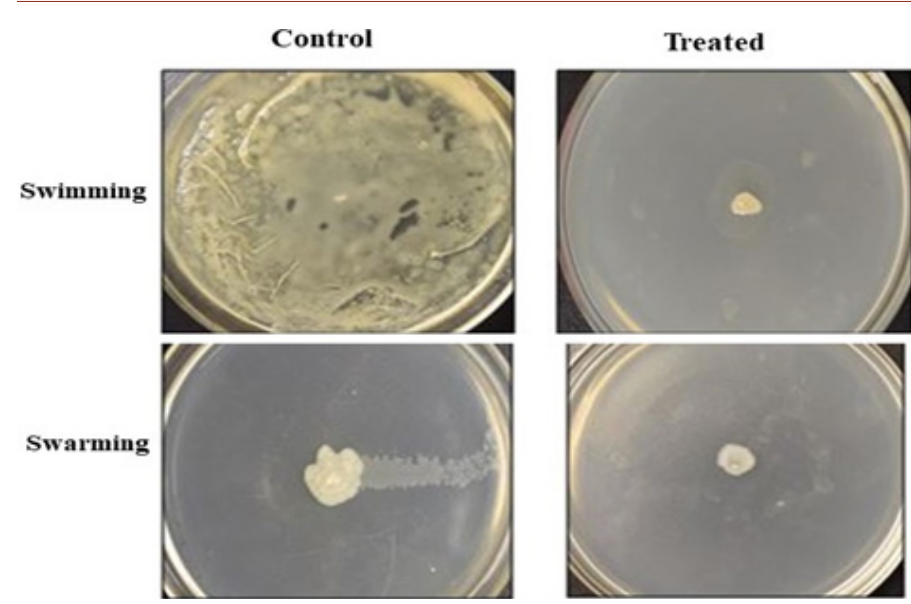

Figure 9: Inhibitory effect of ag nanoparticle on swimming and swarming Motility of Vibrio parahemolyicus.

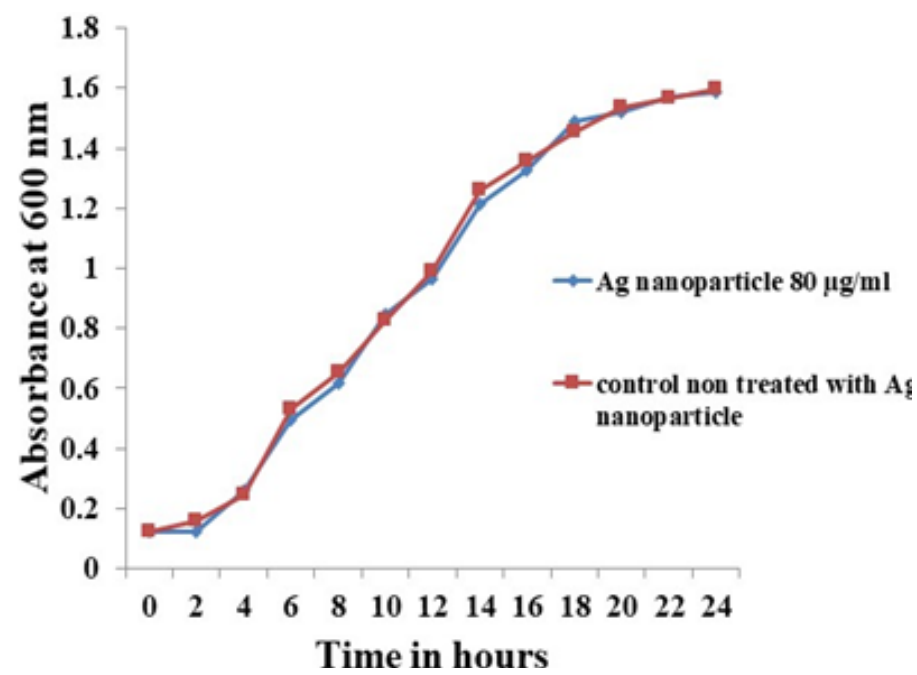

Figure 10: Comparative Growth curve analysis of Vibrio parahemolyicus on treatment with ag nanoparticle and control.

\section{DISCUSSION}

Biofilm formation and antibiotic resistance are the major challenge and pose a serious threat in the pharmaceutical industry. ${ }^{19}$ Besides the clinical pathogen, another major environment strongly affected is aquaculture, over-usage and antibiotic deposition lead to resistance, especially biofilm-assisted pathogens act as a great risk to aquatic animals, as these biofilms function as a reservoir of pathogens that cause several complications for aquatic animals life. Identifying an alternative and effective method to control virulence and biofilm is therefore a big problem. Therefore, the present study empathizes with Ag nanoparticles that attenuate the virulence of Vibrio parahemolyticus, one of the dreadful pathogens in aquatic animals and human. The results are similar to those of silver nanoparticles inhibiting the biofilm and virulence in methicillin-resistant Staphylococcus aureus. ${ }^{20}$ And also few reports with other nanoparticles like $\mathrm{ZnO}$ involved in quorum detection virulence and biofilm reduction were studied. ${ }^{21}$ Quorum sensing induces the virulence of the pathogen through biofilm formation and it is also known that EPS, CSH and motility help in biofilm formation..$^{22}$ In fact, EPS is an essential phenomenon in biofilm formation, the current study also confirms the scenario in which Ag nanoparticles treated with Vibrio parahemolyicus showed a notable decrease in biofilm formation by $63 \%$,

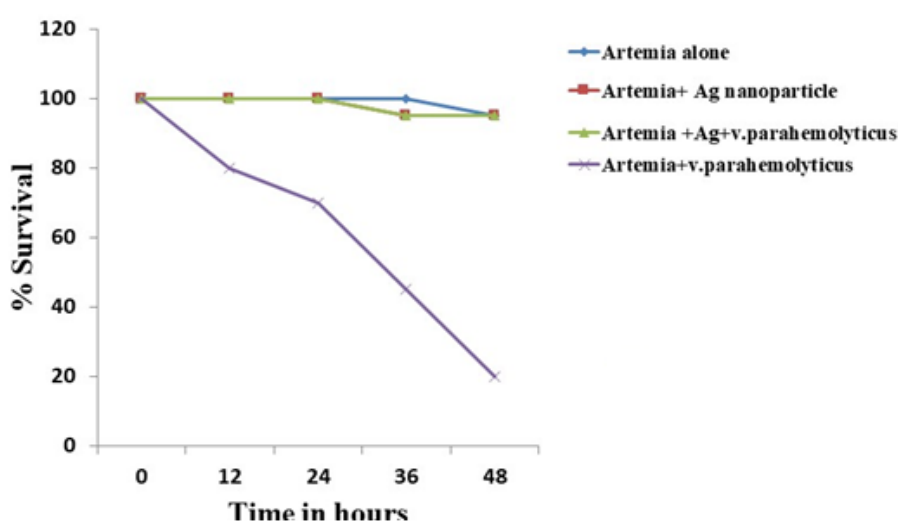

Figure 11: Effect of ag nanoparticle on survival rate of brine shrimp Artemia infected with Vibrio parahemolyticus.

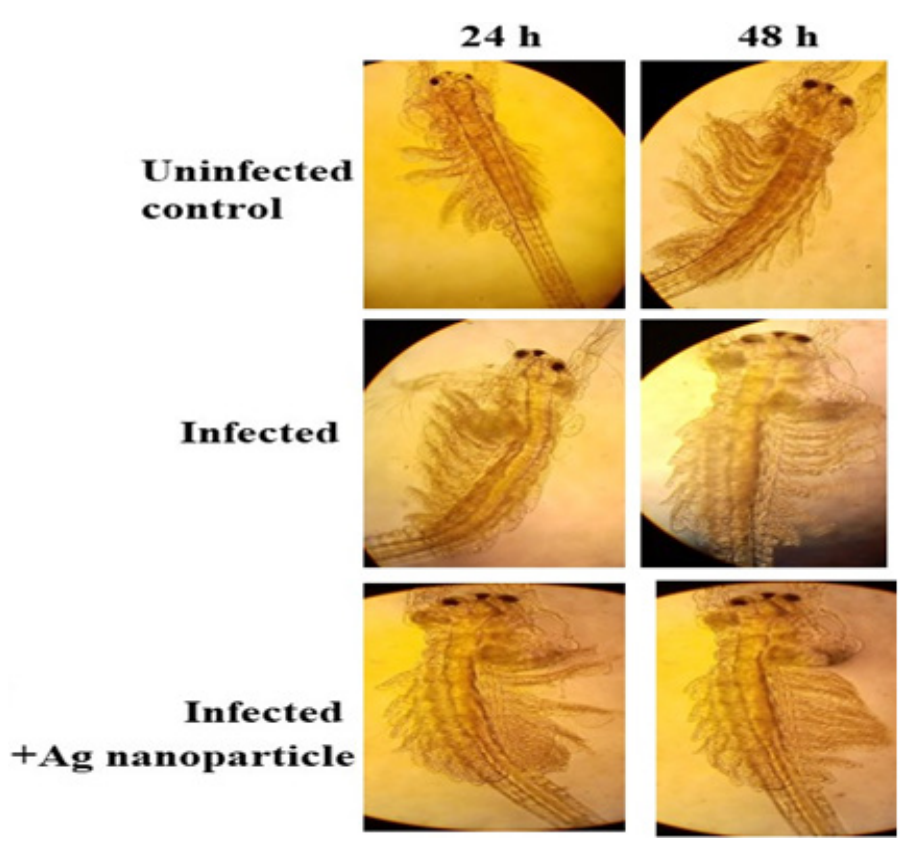

Figure 12: Light microscopic analysis of brine shrimp on treatment with $\mathrm{Ag}$ nanoparticle and control on infection with Vibrio parahemolyticus.

the EPS of 53\% and a 3-fold decrease in CSH followed by reduction in motility, which is similar to the results of Synetochoccus extract inhibiting biofilm-mediated virulence in the Vibrio harveyi and vibrio vulnificus. ${ }^{23}$ Thus, in vitro studies confirm the effectiveness of Ag nanoparticles in inhibiting the virulence cascade in Vibrio parahemolyticus, which thereby in vivo studies were performed on aquatic model brine shrimp were challenged with Vibrio parahemolyticus and the survival rate of Ag nanoparticle treated brine shrimp has increased drastically, 7-fold increased survival rate was observed which clearly authenticate its efficiency as an virulence factor inhibitor and protect the brine shrimp from infection thus exhibiting an proficient use as anti-infective drug in aquatic animals.

\section{CONCLUSION}

Ag nanoparticle can be a potent quorum sensing inhibitor by controlling the biofilm mediated virulent traits in Vibrio parahemolyticus which could possibly combat the Vibriosis in aquatic environment and a great alternative to control antibiotic resistant strain in aquatic environment. However, their exact mechanism is unknown still yet to be investigated. 


\section{ACKNOWLEDGEMENT}

The authors are extremely thankful to the Department of Microbiology, Pondicherry University, Puducherry.

\section{CONFLICT OF INTEREST}

All the authors declare no conflict of interest.

\section{ABBREVIATIONS}

MIC: Minimum Inhibitory Concentration; SEM: Scanning Electron Microscope; EPS: Exopolysaccharide; CSH: Cell Surface Hydrophobicity; BATH: Bacterial Adhesion to Hydrocarbons; Ag: Silver; mM: Milli Molar; RPM: Rotation per minute; HRTEM: High Resolution Transmission Electron Microscope; SAED: Selected Area Electron Diffraction; STEM: Scanning Transmission Electron Microscope; MBC: Minimum Bactericidal Concentration; CFU: Colony Forming Unit; OD: Optical Density.

\section{REFERENCES}

1. Fakruddin M, Hossain Z, Afroz H. Prospects and applications of nanobiotechnology: a medical perspective. J Nanobiotechnology. 2012;10(1):1-8.

2. Handoko CT, Huda A, Gulo F. Synthesis pathway and powerful antimicrobial properties of silver nanoparticle: A critical review. Asian J Sci Res. 2019;12:1-7.

3. Soleimani M, Habibi-Pirkoohi M. Biosynthesis of silver nanoparticles using Chlorella vulgaris and evaluation of the antibacterial efficacy against Staphylococcus aureus. Avicenna J Med Biotechnol. 2017;9(3):120.

4. Patel V, Berthold D, Puranik P, Gantar M. Screening of cyanobacteria and microalgae for their ability to synthesize silver nanoparticles with antibacterial activity. Biotechnol Rep. 2015;5:112-9.

5. Singh RK, Tiwari SP, Rai AK, Mohapatra TM. Cyanobacteria: An emerging source for drug discovery. J Antibiot Res. 2011;64(6):401-12.

6. Chakraborty B, Jayaswal RP, Pankaj PP. Antimicrobial Activity of Spirulina platensis Extract against Gram Positive and Gram Negative Bacteria: A Comparative Study. Int J Curr Pharm. 2015;6(4):212-4.

7. Haendiges J, Timme R, Allard MW, Myers RA, Brown EW, Gonzalez-Escalona N. Characterization of Vibrio parahaemolyticus clinical strains from Maryland (2012-2013) and comparisons to a locally and globally diverse V. parahaemolyticus strains by whole-genome sequence analysis. Front Microbiol. 2015;6:125.

8. Satish L, Santhakumari S, Gowrishankar S, Pandian SK, Ravi AV, Ramesh M. Rapid biosynthesized AgNPs from Gelidiella acerosa aqueous extract mitigates quorum sensing mediated biofilm formation of Vibrio species-an in vitro and in vivo approach. Environ Sci Pollut Res. 2017;24(35):27254-68.

9. Kannan RR, Stirk WA, Staden JV. Synthesis of silver nanoparticles using the seaweed Codium capitatum PC Silva (Chlorophyceae). S Afr J Bot. 2013;86:1-4.
10. Humphries RM, Ambler J, Mitchell SL, Castanheira M, Dingle T, Hindler JA, et al. CLSI methods development and standardization working group best practices for evaluation of antimicrobial susceptibility tests. J Clin Microbiol. 2018;56(4).

11. Packiavathy IA, Priya S, Pandian SK, Ravi AV. Inhibition of biofilm development of uropathogens by curcumin-an anti-quorum sensing Agent from Curcuma longa. Food Chem. 2014; 148:453-60.

12. Sarkar R, Chaudhary SK, Sharma A, Yadav KK, Nema NK, Sekhoacha M, et al. Anti-biofilm activity of Marula-a study with the standardized bark extract. J Ethnopharmacol. 2014;154(1):170-5.

13. Gowrishankar S, Sivaranjani M, Kamaladevi A, Ravi AV, Balamurugan K, Karutha Pandian S. Cyclic dipeptide cyclo (I-leucyl-I-prolyl) from marine Bacillus amyloliquefaciens mitigates biofilm formation and virulence in Listeria monocytogenes. Pathog Dis. 2016;74(4).

14. Courtney HS, Ofek I, Penfound T, Nizet V, Pence MA, Kreikemeyer B, et al. Relationship between expression of the family of $\mathrm{M}$ proteins and lipoteichoic acid to hydrophobicity and biofilm formation in Streptococcus pyogenes. PLoS One. 2009;4(1):e4166.

15. Whitaker WB, Richards GP, Boyd EF. Loss of sigma factor RpoN increases intestinal colonization of Vibrio parahaemolyticus in an adult mouse model. Infect Immu. 2014;82(2):544-56.

16. Defoirdt T, Benneche T, Brackman G, Coenye T, Sorgeloos P, Scheie AA. A quorum sensing-disrupting brominated thiophenone with a promising therapeutic potential to treat luminescent vibriosis. PloS one. 2012;7(7):e41788.

17. Brackman G, Defoirdt T, Miyamoto $C$, Bossier $P$, Calenbergh SV, Nelis H, et al. Cinnamaldehyde and cinnamaldehyde derivatives reduce virulence in vibrio spp. by decreasing the DNA-binding activity of the quorum sensing response regulator LuxR. BMC Microbiol. 2008;8(1):149.

18. Packiavathy IA, Sasikumar P, Pandian SK, Ravi AV. Prevention of quorum-sensing-mediated biofilm development and virulence factors production in vibrio spp. by curcumin. Appl Microbiol Biotechnol. 2013;97(23):10177-87.

19. Nair GB, Ramamurthy T, Bhattacharya SK, Dutta B, Takeda Y, et al. Global dissemination of Vibrio parahaemolyticus serotype O3: K6 and its serovariants. Clin Microbiol Rev. 2007;20(1):39-48.

20. Hamida RS, Ali MA, Goda DA, Khalil MI, Al-Zaban MI. Novel biogenic silver nanoparticle-induced reactive oxygen species inhibit the biofilm formation and virulence activities of methicillin-resistant Staphylococcus aureus (MRSA) strain. Front Bioeng Biotechnol. 2020;8.

21. García-Lara B, Saucedo-Mora MÁ, Roldán-Sánchez JA, Pérez-Eretza B, Ramasamy $M$, Lee J, et al. Inhibition of quorum-sensing-dependent virulence factors and biofilm formation of clinical and environmental Pseudomonas aeruginosa strains by ZnO nanoparticles. Lett Appl Microbiol. 2015;61(3):299-305.

22. Satish L, Santhakumari S, Gowrishankar S, Pandian SK, Ravi AV, Ramesh M Rapid biosynthesized AgNPs from Gelidiella acerosa aqueous extract mitigates quorum sensing mediated biofilm formation of Vibrio species-an in vitro and in vivo approach. Environ Sci Pollut Res. 2017;24(35):27254-68.

23. Santhakumari S, Kannappan A, Pandian SK, Thajuddin N, Rajendran RB, Ravi AV. Inhibitory effect of marine cyanobacterial extract on biofilm formation and virulence factor production of bacterial pathogens causing vibriosis in aquaculture. J Appl Phycol. 2016;28(1):313-24.

Article History: Submission Date : 27-09-2020; Revised Date : 15-10-2020; Acceptance Date : 07-11-2020.

Cite this article: Rathna K, Navyaka, Haque E. Bioengineered Silver Nanoparticle from Spirulina platensis in Attenuating Biofilm Mediated Virulence in Vibrio parahemolyticus: An in vitro and in vivo Approach. Int. J. Pharm. Investigation, 2020;10(4):486-91. 